\title{
ON THE CONVERGENCE OF HILL'S METHOD
}

\author{
CHRISTOPHER W. CURTIS AND BERNARD DECONINCK
}

\begin{abstract}
Hill's method is a means to numerically approximate spectra of linear differential operators with periodic coefficients. In this paper, we address different issues related to the convergence of Hill's method. We show the method does not produce any spurious approximations, and that for selfadjoint operators, the method converges in a restricted sense. Furthermore, assuming convergence of an eigenvalue, we prove convergence of the associated eigenfunction approximation in the $L^{2}$-norm. These results are not restricted to selfadjoint operators. Finally, for certain selfadjoint operators, we prove that the rate of convergence of Hill's method to the least eigenvalue is faster than any polynomial power.
\end{abstract}

\section{INTRODUCTION}

In this paper we study a numerical method, henceforth called Hill's method, used to approximate the spectrum of the operator

$$
S_{p} \psi \equiv \partial_{x}^{p} \psi+\sum_{k=0}^{p-1} f_{k}(x) \partial_{x}^{k} \psi
$$

where $\psi$ is in some appropriate space to be defined later. The coefficient functions $f_{k}(x)$ are smooth, $T$-periodic functions: $f_{k}(x+T)=f_{k}(x), k=0, \ldots, p-1$. This is denoted as

$$
f_{k} \in \mathbf{C}^{\infty}\left(\mathrm{S}_{T}\right) .
$$

Using Floquet and Fourier theory, our approximation starts by computing a bi-infinite matrix representing a parameter-dependent symbol of $S_{p}$. We make the problem finite dimensional by truncating the bi-infinite matrix in both rows and columns; we then compute the eigenvalues of the resulting finite-dimensional matrix. Such an approach is commonly used. This is made more precise in the following section. In modern terminology, this truncation may be called a Galerkin approximation [2, though it is also called a projection method in [3].

In its full generality, Hill's method was first developed in [6]. However, the method appeared in more specialized contexts as early as 1886, when George Hill published [10]. This paper detailed his investigations into the reduced three-body problem, where an analysis of small perturbations led him to seek solutions to the linear problem

$$
\frac{d^{2} \psi}{d x^{2}}+\left(\theta_{0}+2 \sum_{n=1}^{\infty} \theta_{n} \cos (2 n x)\right) \phi=0
$$

Received by the editor October 7, 2008 and, in revised form, February 13, 2009.

2000 Mathematics Subject Classification. Primary 34L16, 65L07.

(C)2009 American Mathematical Society 
Here $\theta_{k}, k=1,2, \ldots$, are real parameters. In his analysis, Hill incorporated both Floquet and Fourier theory, which led him to consider infinite-dimensional matrices and their corresponding determinants. Hill used these determinants in a formal way, and he attempted to approximate the spectra of the infinite-dimensional matrices using the spectra of three-by-three truncations. Inspired by Hill's work, a rigorous theory on determinants of infinite matrices was initiated by Poincaré [17] and von Koch 22]. This in turn has led to a modern theory of determinants of operators defined over Banach spaces. The treatise by Gohberg, Goldberg, and Krupnick [9] provides an excellent introduction to both the classical origins and modern developments of infinite-dimensional determinants, and our work relies heavily on the material in [9] (see also 8] and 4]). However, we do not develop this theory any further.

Instead, we focus on proving the validity of Hill's truncation. This problem, in turn, has its own deep and storied history. A wonderful introduction can be found in [3]. Likewise, in the same reference, one can find a number of examples where using finite-dimensional approximations to compute the spectra of infinitedimensional operators fails spectacularly. For our problem, however, we show that for general $S_{p}$, Hill's method never converges to spurious eigenvalues in compact domains. In the case that $S_{p}$ is selfadjoint, we go further and show, again on any compact domain, that Hill's method converges to the spectrum of $S_{p}$ restricted to said domain. Furthermore, assuming the convergence of an approximate sequence of eigenvalues to a simple eigenvalue, we show that the corresponding eigenvector approximations converge to a true eigenvector in the $L^{2}$-norm.

As shown in [6], Hill's method is exact for constant-coefficient problems. By restricting ourselves to a particular class of selfadjoint operators, which represent the simplest case of non-constant coefficient equations, we show Hill's method approximates the smallest eigenvalue faster than any polynomial power. This restricted class of operators includes classic problems such as Mathieu's equation, and it represents a non-trivial and interesting body of problems for which Hill's method is an excellent approximation scheme.

Another, more abstract but also more general, approach for analyzing Hill's method can be found in the notes of G.M. Vainikko (Chapter 4 of [13]). This approach applies to a more general class of problems than just Hill's method, and once the approach is mastered, its application to Hill's method can be viewed as a corollary. The results in [13] not only allow for establishing the convergence of Hill's method, but the rate of convergence can also be determined. The rate thus found is identical to the one we establish in this paper. In the case of symmetric operators, a convergence proof and rate can also be found in [7. However, the class of operators considered in [7] is far more restricted than in this paper or [13]. Furthermore, the rate of convergence obtained is far slower than what we or [13] are able to show.

The key to the deeper results in 13 is the notion of the aperture between subspaces of a Banach space (see also Chapter 4 of [12]). We make no use of this idea, or any other result found in 13. Instead, we use a more direct and explicit approach, which may be more natural or intuitive if one is interested in Hill's method in its own right, as opposed to regarding it as a special case of a more general problem. Indeed, as mentioned above, Hill's method led us to consider determinants 
of infinite-dimensional operators and the work of [9]. Thus, the methods presented in this paper are new and hopefully insightful.

On a final note, our manuscript contains neither numerical illustrations of our results nor examples where the method fails. There are two reasons for this. First, many examples have already been demonstrated in 4. Second, our results are positive results about the validity of Hill's truncation. This implies the non-existence of numerical counterexamples to what we prove, hence precluding any numerical illustrations of the method's failure.

Remark. - The form of the operator (11) is restrictive in that we equate the coefficient of the highest-order derivative to one. Were the coefficient a constant, this would not change our results. The effect of a non-constant coefficient on our work is non-trivial. However, in many problems (linear stability, scattering) the spectral problems that arise are of the form used here (see the examples in [6]), although variations occur.

- Numerically computing the eigenvalues of a matrix is a non-trivial problem. It is not a problem we consider in this paper. Our sole interest is in the relation between the finite-dimensional approximations as obtained through Hill's method and the problem they are meant to approximate.

- The work in this paper focuses on spectral problems defined by scalar differential operators (11). This restriction is made for ease of presentation. Hill's method, in essence a Galerkin method, works equally well for systems of equations or for problems with multiple independent variables [6]. Our methods of analysis used apply to the system case, but modifications are necessary for the multi-dimensional case.

- Combining the ideas of Floquet decomposition and the truncation of matrix representations of operators is frequently done when considering periodic operator equations. Three contemporary examples of this can be found in [19], 23], and [24. Special mention should be made with regards to [24].

The outline of this paper is as follows. In the next section we present the details of the method for the scalar problem. That section is also used to settle various notational issues. Section 3 presents our proofs relating to the convergence of Hill's method. We break the section into two parts, the first dealing with non-selfadjoint operators, the second dealing with selfadjoint operators in particular. Section 4 contains our proof of the convergence of the associated eigenvector approximations. Again we emphasize that this result holds for general operators. Next, Section 5 presents our proof of spectral convergence for the least eigenvalue. Finally, the last section summarizes our findings.

\section{HILL'S METHOD}

Hill's method is discussed in great detail in [6]. In essence the method combines a Floquet (or Bloch) decomposition with a Fourier expansion so as to reduce the numerical computation of the spectrum of a periodic differential operator to the computation of spectra of a family of (finite-dimensional) matrices. Before we continue, we define some relevant spaces that will be used throughout the rest of the paper. Let $\mathrm{L}_{2}\left(\mathrm{~S}_{T}\right)$ be defined as the completion of $\mathrm{C}\left(S_{T}\right)$, the space of $T$-periodic, 
continuous functions, with respect to the $L^{2}$-norm on the interval $[-T / 2, T / 2]$. Let

$$
\mathrm{e}_{n}(x)=\frac{e^{-i 2 \pi n x / T}}{\sqrt{T}}, n \in \mathbb{Z},
$$

so that for $\phi \in \mathrm{L}_{2}\left(\mathrm{~S}_{T}\right)$, we have the associated Fourier series

$$
\phi(x)=\sum_{n=-\infty}^{\infty} \hat{\phi}_{n} \mathrm{e}_{n}(x)
$$

with

$$
\hat{\phi}_{n}=\left\langle\phi, \mathrm{e}_{n}\right\rangle=\frac{1}{\sqrt{T}} \int_{-T / 2}^{T / 2} \phi(x) \mathrm{e}_{n}^{*}(x) d x .
$$

This allows us to associate with every function $\phi \in \mathrm{L}_{2}\left(\mathrm{~S}_{T}\right)$ its Fourier transform

$$
\hat{\phi} \equiv\left\{\hat{\phi}_{n}\right\}_{n=-\infty}^{\infty} .
$$

We define the Sobolev spaces $\mathrm{H}^{p}\left(\mathrm{~S}_{T}\right)$ in a similar fashion, and in our paper, we define the norm on $\mathrm{H}^{p}\left(\mathrm{~S}_{T}\right)$ as $([2]$, p. 308)

$$
\|\phi\|_{2, p}^{2} \equiv\left|\hat{\phi}_{0}\right|^{2}+\sum_{|k|>0}\left(\frac{2 \pi k}{T}\right)^{2 p}\left|\hat{\phi}_{k}\right|^{2} \text {. }
$$

The Floquet-Bloch decomposition. First, we define $S_{p}$ over the Sobolev space $\mathrm{H}^{p}(\mathbb{R})$, with

$$
\mathrm{H}^{p}(\mathbb{R})=\left\{\left.f \in \mathrm{L}_{2}(\mathbb{R})\left|\sum_{k=0}^{p} \int_{\mathbb{R}}\right| f^{k}(x)\right|^{2} d x<\infty\right\},
$$

where $f^{k}$ denotes the $k^{t h}$ weak derivative of $f$. This makes $S_{p}$ closed and densely defined. We can likewise turn the operator $S_{p}-\lambda$ into a first-order differential operator defined on $\mathrm{H}^{1}\left(\mathbb{R} ; \mathbb{C}^{p}\right)$, where the notation means that the space $\mathrm{H}^{1}\left(\mathbb{R} ; \mathbb{C}^{p}\right)$ consists of $\mathbb{C}^{p}$-valued functions with one weak derivative, and for which the function and its derivate have $\mathbb{C}^{p}$-norms that are both in $\mathrm{L}_{2}(\mathbb{R})$ (see 18 for more details). Denote the first-order differential operator as $S(x ; \lambda)=\frac{d}{d x}-B(x ; \lambda)$, where $B(x ; \lambda)$ is a $p \times p$ matrix. By definition,

$$
\sigma\left(S_{p}\right)=\{\lambda \in \mathbb{C}: S(x ; \lambda) \text { does not have a bounded inverse }\} .
$$

Following [20], we use the following decomposition of $\sigma\left(S_{p}\right)$ (see also [5], [16]).

- $\sigma_{p t}\left(S_{p}\right)=\{\lambda \in \mathbb{C}: S(x ; \lambda)$ is Fredholm with zero index. $\}$

- $\sigma_{\text {ess }}\left(S_{p}\right)=\sigma\left(S_{p}\right) \backslash \sigma_{p t}\left(S_{p}\right)$.

Since $S_{p}$ has only periodic coefficients, we need only compute $\sigma_{\text {ess }}\left(S_{p}\right)$ [20]. This reduces to the following problem.

Theorem 1. $\lambda \in \sigma\left(S_{p}\right)$ if and only if the differential equation

$$
\begin{aligned}
\frac{d u}{d x} & =B(x ; \lambda) u, 0<x<T, \\
u(T) & =e^{i \mu T} u(0)
\end{aligned}
$$

has a solution for some $\mu \in[0,2 \pi / T)$.

Proof. See [20, page 1001. 
We transform the differential equation in Theorem 1 into

$$
\begin{aligned}
\frac{d \psi}{d x} & =\tilde{B}(x ; \lambda, \mu) \psi, 0<x<T, \\
\psi(T) & =\psi(0)
\end{aligned}
$$

via the transformation $\psi(x)=e^{-i \mu x} u(x)$. Note that $\tilde{B}(x ; \lambda, \mu)=B(x ; \lambda)-i \mu$. We can then restate Theorem 1 as

Theorem 2. $\lambda \in \sigma\left(S_{p}\right)$ if and only if the differential equation

$$
\frac{d \psi}{d x}=\tilde{B}(x ; \lambda, \mu) \psi, \psi \in H^{1}\left(S_{T} ; \mathbb{C}^{p}\right)
$$

has a solution for some $\mu \in[0,2 \pi / T)$.

It is easy to show that the $p^{t h}$-order system in Theorem 2 is equivalent to the scalar problem

$$
S_{p}^{\mu} \phi=\lambda \phi, \phi \in \mathrm{H}^{p}\left(\mathrm{~S}_{T}\right)
$$

where

$$
S_{p}^{\mu} \phi=\mathrm{e}^{-i \mu x} S_{p}\left(\mathrm{e}^{i \mu x} \phi\right) .
$$

An explicit form for $S_{p}^{\mu}$ is found in [6]. Theorem 2] implies that we can write $\sigma\left(S_{p}\right)$ as

$$
\sigma\left(S_{p}\right)=\bigcup_{\mu} \sigma\left(S_{p}^{\mu}\right)
$$

As implied by (14), for each value of $\mu, \sigma\left(S_{p}^{\mu}\right)$ consists only of point spectra. We approximate these point sets numerically for a fixed value of $\mu$.

The Fourier decomposition. To reduce the problem to linear algebra, we resort to a Galerkin method [2] using the orthonormal basis $\mathrm{e}_{n}$ given at the beginning of this section. Of course, given any orthonormal basis $\left\{\varphi_{j}\right\}$, we can generate a matrix representation for any linear operator $M$ with entries $\left\langle M \varphi_{j}, \varphi_{k}\right\rangle,(j, k) \in \mathbb{Z}^{2}$. Our particular choice of basis reflects the boundary conditions of our eigenvalue problem (14). We interchangeably refer to the bi-infinite matrix, with entries $\left\langle S_{p}^{\mu} \mathrm{e}_{j}, \mathrm{e}_{k}\right\rangle$, as the Fourier transform or symbol of the linear operator $S_{p}^{\mu}$. We denote the symbol (or Fourier transform, or bi-infinite matrix representation) of $S_{p}^{\mu}$ as $\hat{S}_{p}^{\mu}$, where the $(n, m)^{t h}$ entry of $\hat{S}_{p}^{\mu}$ is denoted by $\hat{S}_{p, n m}^{\mu}=\left\langle S_{p}^{\mu} \mathrm{e}_{m}, \mathrm{e}_{n}\right\rangle$. We write the Fourier transform of our eigenvalue problem (14) as

$$
\hat{S}_{p}^{\mu} \hat{\phi}=\lambda \hat{\phi} .
$$

Finite-dimensional projection. The last step of Hill's method requires the introduction of the orthogonal projection operator $P_{N}$ onto the subspace spanned by the Fourier modes from $-N$ to $N$. The effect of $P_{N}$ applied to a periodic function is truncation of the Fourier series, i.e.,

$$
P_{N} \phi(x)=\sum_{n=-N}^{N} \hat{\phi}_{n} \mathrm{e}_{n}(x) .
$$


Likewise, the action of the symbol of $P_{N}, \hat{P}_{N}$, will give

$$
\left(\hat{P}_{N} \hat{\phi}\right)_{n}=\left\{\begin{array}{cc}
0, & |n|>N \\
\hat{\phi}_{n}, & |n| \leq N .
\end{array}\right.
$$

Define the $(2 N+1) \times(2 N+1)$ matrix $\hat{S}_{N}^{\mu, \tau}$ via

$$
\hat{P}_{N} \hat{S}_{p}^{\mu} \hat{P}_{N}=\left(\begin{array}{ccccc}
\ddots & & \vdots & & \cdots \\
& 0 & 0 & 0 & \\
\cdots & 0 & \hat{S}_{N}^{\mu, \tau} & 0 & \cdots \\
& 0 & 0 & 0 & \\
\cdots & & \vdots & & \ddots
\end{array}\right) \text {, }
$$

where the $\tau$ emphasizes that $\hat{S}_{N}^{\mu, \tau}$ is a truncation of a bi-infinite matrix. As a matter of convention, for any operator $A$ with symbol $\hat{A}$, we define $\hat{A}_{N}^{\tau}$ in the same fashion, namely

$$
\hat{P}_{N} \hat{A} \hat{P}_{N}=\left(\begin{array}{ccccc}
\ddots & & \vdots & & \cdots \\
& 0 & 0 & 0 & \\
\cdots & 0 & \hat{A}_{N}^{\tau} & 0 & \cdots \\
& 0 & 0 & 0 & \\
\cdots & & \vdots & & \ddots
\end{array}\right) .
$$

Likewise we introduce the shorthand $\hat{A}_{N}=\hat{P}_{N} \hat{A} \hat{P}_{N}$.

Finally, we define the approximate eigenvalue problem

$$
\hat{S}_{N}^{\mu, \tau} \hat{\phi}_{N}^{\tau}=\lambda_{N} \hat{\phi}_{N}^{\tau}
$$

where the subscript $N$ on $\lambda_{N}$ reinforces the order of the approximation. A more detailed derivation is presented in $[\underline{6}$.

\section{Proof of Convergence}

By the convergence of Hill's method, we mean that the following two properties are satisfied.

(1) For a given sequence $\left\{\lambda_{N}\right\}_{N=1}^{\infty}, \lambda_{N} \in \sigma\left(\hat{S}_{N}^{\mu, \tau}\right)$, and for any $\epsilon>0$, there exists an integer $M$ such that any $\lambda_{N}, N \geq M$, is in an $\epsilon$-neighborhood of some $\lambda \in \sigma\left(S_{p}^{\mu}\right)$.

(2) For all $\lambda \in \sigma\left(S_{p}^{\mu}\right)$, there exists some sequence $\left\{\lambda_{N}\right\}_{N=1}^{\infty}, \lambda_{N} \in \sigma\left(\hat{S}_{p N}^{\mu}\right)$, such that $\lambda_{N} \rightarrow \lambda$.

The first condition ensures that Hill's method is accurate, but it leaves open the possibility that the method may not produce all of $\sigma\left(S_{p}^{\mu}\right)$. Likewise, the second statement ensures that the method will faithfully reproduce all of $\sigma\left(S_{p}^{\mu}\right)$, but it does not rule out that the method will produce spurious information. It is this distinction that leads us to refer to the first condition as the "no-spurious modes" condition. 
We are able to prove a slightly restricted version of the no-spurious modes condition for any operator $S_{p}^{\mu}$. We modify the condition only by requiring the arbitrary sequence $\left\{\lambda_{N}\right\}_{N=1}^{\infty}$ to be confined to a compact subset of the complex plane. The second condition is essentially proved in [18, for selfadjoint operators. We have not been able to improve upon this restriction. However, we present the outline of the proof provided in [18, for the sake of completeness.

3.1. Proof of the no-spurious-modes condition. Our proof of the first condition relies upon one major theorem. Before proving this theorem, we need to develop and explain the basic machinery necessary for our proof. First, for notational ease, we define the operator $S^{1}$ as

$$
\text { - } \mathrm{D}\left(S^{1}\right)=\mathrm{H}^{p}\left(\mathrm{~S}_{T}\right), S^{1} \phi=S_{p}^{\mu} \phi \text {. }
$$

We now provide a brief introduction to the theory of determinants of operators on a separable Hilbert Space, say H. This material was developed in 9, and we reproduce it here only for completeness or to clarify some points made in 9 . Let $\mathrm{B}(\mathrm{H})$ denote the space of all bounded operators from $\mathrm{H}$ into itself. Let $\mathrm{F}$ denote the space of finite-rank operators. For our purposes, it is not sufficient to use the operator norm induced by the norm on $\mathrm{H}$, say $\|\cdot\|$. Instead, we need to introduce a new norm $\|\cdot\|_{Z}$, where $\mathrm{Z}$ denotes a subalgebra of $\mathrm{B}(\mathrm{H})$ such that $\mathrm{F} \cap \mathrm{Z}$ is dense in $\mathrm{Z}$ and

$$
\|\cdot\| \leq \mathrm{C}\|\cdot\|_{\mathrm{Z}}
$$

where $\mathrm{C}$ is a constant. Thus $\mathrm{Z}$ is an embedded subalgebra in $\mathrm{B}(\mathrm{H})$. Likewise, if the space of finite-rank operators is dense in $Z$, this implies every element in $Z$ is compact. Next, define the trace of $K \in \mathrm{F} \cap \mathrm{Z}$ by

$$
\operatorname{tr}(K)=\sum_{k=1}^{n} \lambda_{k}
$$

and define the determinant of $I+K$ as

$$
\operatorname{det}(I+K)=\prod_{k=1}^{n}\left(1+\lambda_{k}\right)
$$

where $n$ is the rank of $K$ and $\lambda_{k}$ are the eigenvalues of $K$.

The issue at hand is whether we can find some continuous function that will serve as an extension of the determinant, which has only been defined on $\mathrm{F} \cap \mathrm{Z}$. A necessary and sufficient condition for this (see [9]) is if the trace is a bounded linear functional in the Z-norm, i.e.,

$$
|\operatorname{tr}(K)| \leq M\|K\|_{\mathrm{Z}}
$$

holds for all $K \in \mathrm{F} \cap \mathrm{Z}$, where $M$ is a constant independent of $K$. If this condition holds, then for $K \in \mathrm{Z}$, we know there exists a sequence of finite-rank operators $K_{N}$ such that

$$
\lim _{N \rightarrow \infty}\left\|K_{N}-K\right\|_{\mathrm{Z}}=0,
$$

and we can define the Z-determinant of $I+K$ as

$$
\operatorname{det}_{\mathrm{Z}}(I+K)=\lim _{N \rightarrow \infty} \operatorname{det}\left(I+K_{N}\right) .
$$

Using the above definitions, one can prove [9]: 
Theorem 3. $(I+K)^{-1}$ exists if and only if $\operatorname{det}_{\mathrm{Z}}(I+K) \neq 0$.

A space well suited for our purposes was developed by Gohberg et al. 9]. Define the subalgebra $\Omega$ via:

$$
\Omega \equiv\left\{A \in B\left(\mathrm{~L}_{2}\left(\mathrm{~S}_{T}\right)\right): \max \left(\lim _{M \rightarrow \infty}\left|\sum_{n=-M}^{M} \hat{A}_{n n}\right|,\left(\sum_{n, m=-\infty}^{\infty}\left|\hat{A}_{n m}\right|^{2}\right)^{1 / 2}\right)<\infty\right\} .
$$

For $A \in \Omega$, we have the corresponding norm $\|A\|_{\Omega}$ defined by

$$
\|A\|_{\Omega} \equiv \max \left(\left|\lim _{M \rightarrow \infty} \sum_{n=-M}^{M} \hat{A}_{n n}\right|,\left(\sum_{n, m=-\infty}^{\infty}\left|\hat{A}_{n m}\right|^{2}\right)^{1 / 2}\right)
$$

where $\hat{A}_{n m}=\left\langle A \mathrm{e}_{m}, \mathrm{e}_{n}\right\rangle$, and $\mathrm{e}_{n}$ is defined as in (4). If $A \in \Omega$, we see that

$$
\|A \phi\|_{2}^{2}=\sum_{n=-\infty}^{\infty}\left|\sum_{m=-\infty}^{\infty} \hat{A}_{n m} \hat{\phi}_{m}\right|^{2} \leq\|\phi\|_{2}^{2} \sum_{n=-\infty}^{\infty} \sum_{m=-\infty}^{\infty}\left|\hat{A}_{n m}\right|^{2}
$$

and therefore $\|A\|_{2} \leq\|A\|_{\Omega}$. The next lemma easily follows.

Lemma 4. For all finite rank operators A, we have

$$
|\operatorname{tr}(A)| \leq\|A\|_{\Omega} .
$$

Proof. Given that $A$ has finite rank, let $\psi_{1}, \ldots, \psi_{n}$ be an orthonormal basis for the range of $A$. Then we may write $A$ as

$$
A=\sum_{k=1}^{n} \psi_{k}\left\langle A \cdot, \psi_{k}\right\rangle
$$

It is clear that

$$
\operatorname{tr}\left(\psi_{k}\left\langle A \cdot, \psi_{k}\right\rangle\right)=\left\langle A \psi_{k}, \psi_{k}\right\rangle,
$$

and therefore

$$
\operatorname{tr}(A)=\sum_{k=1}^{n}\left\langle A \psi_{k}, \psi_{k}\right\rangle
$$

Since $\psi_{k} \in \mathrm{L}_{2}\left(\mathrm{~S}_{T}\right)$, we can expand $\psi_{k}$ as

$$
\psi_{k}=\sum_{j=-\infty}^{\infty} \hat{\psi}_{k j} \mathrm{e}_{j}
$$

Note that

$$
\left\langle A \mathrm{e}_{j}, \mathrm{e}_{j}\right\rangle=\sum_{k=1}^{n} \hat{\psi}_{k j}\left\langle A \mathrm{e}_{j}, \psi_{k}\right\rangle
$$


Therefore we can rewrite the trace of a finite-rank operator as

$$
\begin{aligned}
\operatorname{tr}(A) & =\sum_{k=1}^{n}\left\langle A \psi_{k}, \psi_{k}\right\rangle \\
& =\sum_{k=1}^{n} \sum_{j=-\infty}^{\infty} \hat{\psi}_{k j}\left\langle A \mathrm{e}_{j}, \psi_{k}\right\rangle \\
& =\sum_{j=-\infty}^{\infty} \sum_{k=1}^{n} \hat{\psi}_{k j}\left\langle A \mathrm{e}_{j}, \psi_{k}\right\rangle \\
& =\sum_{j=-\infty}^{\infty}\left\langle A \mathrm{e}_{j}, \mathrm{e}_{j}\right\rangle .
\end{aligned}
$$

Thus from the definition of $\|\cdot\|_{\Omega}$ we have the result.

Similarly, we have

Lemma 5. Every finite-rank operator is in $\Omega$.

Proof. We know that the trace of a finite-rank operator, say $A$, is bounded, and thus from the previous lemma we know that $\left|\sum_{j=-\infty}^{\infty} \hat{A}_{j j}\right|<\infty$. Likewise we have

$$
\begin{aligned}
\sum_{j, k}\left|\left\langle A \mathrm{e}_{j}, \mathrm{e}_{k}\right\rangle\right|^{2} & =\sum_{j, k}\left|\sum_{l=1}^{n} \hat{\psi}_{l k}\left\langle A \mathrm{e}_{j}, \psi_{l}\right\rangle\right|^{2} \\
& \leq\left(\sum_{k} \sum_{l=1}^{n}\left|\hat{\psi}_{l k}\right|^{2}\right)\left(\sum_{j} \sum_{l=1}^{n}\left|\left\langle\mathrm{e}_{j}, A^{\dagger} \psi_{l}\right\rangle\right|^{2}\right) \\
& \leq n \sum_{l=1}^{n} \|\left. A^{\dagger} \psi_{l}\right|_{2} ^{2}<\infty,
\end{aligned}
$$

where $A^{\dagger}$ denotes the adjoint of $A$. Therefore every finite-rank operator is in $\Omega$.

Lastly, we need to show that $\mathrm{F}$ is dense in $\Omega$. We do this, and also establish a useful result for our main theorem, in the following lemma.

Lemma 6. If $A \in \Omega$, then $\lim _{N \rightarrow \infty}\left\|A-P_{N} A P_{N}\right\|_{\Omega}=0$.

Proof. It is clear that

$$
\left\|A-P_{N} A P_{N}\right\|_{\Omega} \leq\left\|\left(I-P_{N}\right) A\right\|_{\Omega}+\left\|P_{N} A\left(I-P_{N}\right)\right\|_{\Omega} .
$$

With

$$
\left\|\left(I-P_{N}\right) A\right\|_{\Omega}=\max \left(\left|\sum_{|n|>N} \hat{A}_{n n}\right|,\left(\sum_{|n|>N} \sum_{m=-\infty}^{\infty}\left|\hat{A}_{n m}\right|^{2}\right)^{1 / 2}\right)
$$

and

$$
\left\|P_{N} A\left(I-P_{N}\right)\right\|_{\Omega}^{2}=\sum_{|n| \leq N} \sum_{|m|>N}\left|\hat{A}_{n m}\right|^{2}
$$

the result follows, since $\|A\|_{\Omega}<\infty$. 
This shows that for $A \in \Omega$, we have

$$
\operatorname{det}_{\Omega}(I+A)=\lim _{N \rightarrow \infty} \operatorname{det}\left(I+P_{N} A P_{N}\right),
$$

where

$$
\operatorname{det}\left(I+P_{N} A P_{N}\right) \equiv \operatorname{det}\left(\hat{I}_{N}^{\tau}+\hat{A}_{N}^{\tau}\right) .
$$

For omitted proofs and more details on this material, the interested reader is advised to consult [9].

Finally, we need two key facts about operators of the form $I+K$, where $I$ is the identity and $K$ is compact.

- $I+K$ is a Fredholm operator.

- $i(I+K)=0$.

Note that for any Fredholm operator $F$,

$$
i(F) \equiv \operatorname{dim}(\operatorname{ker}(F))-\operatorname{dim}\left(\operatorname{ker}\left(F^{\dagger}\right)\right),
$$

where $F^{\dagger}$ again denotes the adjoint of $F$. For the proof, see 21].

With these tools in hand, we prove the following theorem. This theorem will be the engine to drive the proof of the no-spurious-mode condition.

Theorem 7. Let $\gamma \in \rho\left(S^{1}\right)$. Then there exists some constant $M_{\gamma}$ such that for $N \geq M_{\gamma}, \gamma \in \rho\left(\hat{S}_{N}^{1, \tau}\right)$.

Proof. Define the operator $B: \mathrm{L}_{2}\left(\mathrm{~S}_{T}\right) \rightarrow \mathrm{L}_{2}\left(\mathrm{~S}_{T}\right)$ via

$$
(\hat{B} \hat{\psi})_{n}=\left\{\begin{array}{rr}
\left(\frac{2 \pi n i}{T}\right)^{-p} \hat{\psi}_{n}, & n \neq 0, \\
i^{-p} \hat{\psi}_{0}, & n=0,
\end{array}\right.
$$

where $\hat{\psi}_{n}$ are the components of the vector $\hat{\psi} \in l_{2}$, and $\hat{B}$ is the symbol of $B$. $B$, when applied to $S^{1}-\gamma$, is introduced to nullify the growth along the diagonal of $\hat{S}^{1}-\gamma$. Clearly $\mathrm{H}^{p}\left(\mathrm{~S}_{T}\right) \subset \mathrm{R}(B)$. With $\gamma \in \rho\left(S^{1}\right)$, we have that $S^{1}-\gamma$ is a bijection from $\mathrm{H}^{p}\left(\mathrm{~S}_{T}\right)$ to $\mathrm{L}_{2}\left(\mathrm{~S}_{T}\right)$ by definition. Therefore, we define the operator $A$ whose symbol is $\hat{B}\left(\hat{S}^{1}-\gamma\right)$, noting that $\mathrm{H}^{p}\left(\mathrm{~S}_{T}\right) \subset \mathrm{R}(A)$.

Now consider computing the matrix product of $\hat{B}$ and $\left(\hat{S}^{1}-\gamma\right)$. Clearly this operator is the extension of $\hat{A}$, and we will show that it is a bounded operator on $l_{2}$. Therefore, it must be the unique bounded extension of $\hat{A}[18$. We refer to the extension of $\hat{A}$ as $\hat{A}$ to economize on notation. Given that $\delta_{n m}$ is the Kroenecker delta function, the terms of $\hat{A}$ are then

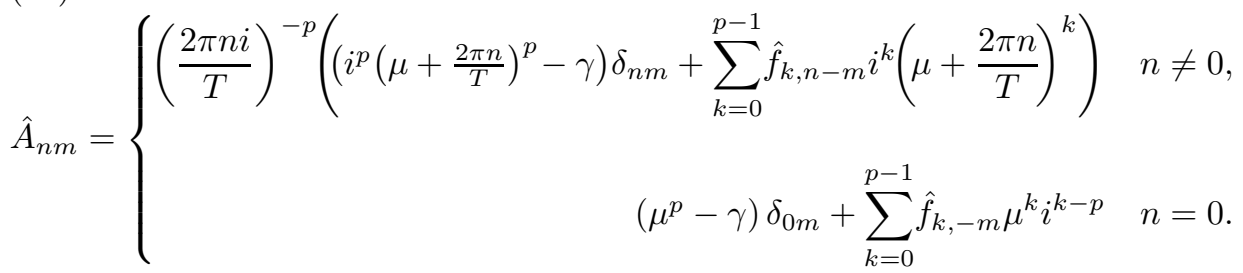

See [6], equation 17, for an explicit derivation. Therefore, for $n \neq 0$,

$$
\hat{A}_{n n}=1+\frac{T}{2 \pi}\left(p \mu-i \hat{f}_{p-1,0}\right) \frac{1}{n}+\mathrm{O}\left(\frac{1}{n^{2}}\right),
$$


which shows that

$$
\lim _{M \rightarrow \infty} \sum_{n=-M}^{M}\left(\hat{A}_{n n}-1\right)<\infty .
$$

Likewise, we have also shown that

$$
\sum_{n=-\infty}^{\infty}\left|\hat{A}_{n n}-1\right|^{2}<\infty
$$

For $n \neq m$ and $n \neq 0$, we have

$$
\begin{aligned}
\left|\hat{A}_{n m}\right|^{2} & \leq\left(\frac{T}{2 \pi n}\right)^{2 p}\left(\sum_{k=0}^{p-1}\left|\hat{f}_{k, n-m}\right|\left|\mu+\frac{2 \pi n}{T}\right|^{k}\right)^{2} \\
& \leq\left(\sum_{k=0}^{p-1}\left|\hat{f}_{k, n-m}\right|^{2}\right)\left(\sum_{k=0}^{p-1}\left(\frac{T}{2 \pi n}\right)^{2 p}\left|\mu+\frac{2 \pi n}{T}\right|^{2 k}\right),
\end{aligned}
$$

while for $n=0$ we have

$$
\left|\hat{A}_{0 m}\right|^{2} \leq \frac{\mu^{2 p}-1}{\mu^{2}-1} \sum_{k=0}^{p-1}\left|\hat{f}_{k,-m}\right|^{2} .
$$

Therefore

$$
\sum_{m \neq n,|n|>0}\left|\hat{A}_{n m}\right|^{2} \leq\left(\sum_{|n|>0} \sum_{k=0}^{p-1}\left(\frac{T}{2 \pi n}\right)^{2 p}\left|\mu+\frac{2 \pi n}{T}\right|^{2 k}\right)\left(\sum_{m=-\infty}^{\infty} \sum_{k=0}^{p-1}\left|\hat{f}_{k, m}\right|^{2}\right) .
$$

The above shows that

$$
\sum_{n, m}\left|\hat{A}_{n m}-\delta_{n m}\right|^{2}<\infty
$$

and therefore $A-I \in \Omega$. Let $K=A-I$, and so $K$ is compact. It is then clear that $A \in \mathrm{B}\left(\mathrm{L}_{2}\left(\mathrm{~S}_{T}\right)\right)$, and that $A$ is Fredholm. Therefore the range of $A$ is closed. We know $\mathrm{H}^{p}\left(\mathrm{~S}_{T}\right) \subset \mathrm{R}(A), \mathrm{H}^{p}\left(\mathrm{~S}_{T}\right)$ is dense in $\mathrm{L}_{2}\left(\mathrm{~S}_{T}\right)$, and so together these facts imply $\mathrm{R}(A)=\mathrm{L}_{2}\left(\mathrm{~S}_{T}\right)$. Hence $\operatorname{dim}\left(\operatorname{ker}\left(A^{\dagger}\right)\right)=0$, and $i(A)=0$, so $\operatorname{dim}(\operatorname{ker}(A))=0$. Therefore $A$ is a bounded bijection from $\mathrm{L}_{2}\left(\mathrm{~S}_{T}\right)$ to $\mathrm{L}_{2}\left(\mathrm{~S}_{T}\right)$, which means $A$ has a bounded inverse by the Open Mapping Theorem.

Knowing that $A$ has a bounded inverse and that $A \in \Omega$, it follows from Theorem 3 that $\operatorname{det}_{\Omega}(A) \neq 0$. We have

$$
\begin{aligned}
\operatorname{det}_{\Omega}(A) & =\lim _{N \rightarrow \infty} \operatorname{det}\left(I+P_{N} K P_{N}\right) \\
& =\lim _{N \rightarrow \infty} \operatorname{det}\left(\hat{I}_{N}^{\tau}+\hat{K}_{N}^{\tau}\right),
\end{aligned}
$$

and thus there exists a constant $M_{\gamma}$ such that for $N \geq M_{\gamma}$, $\operatorname{det}\left(\hat{I}_{N}^{\tau}+\hat{K}_{N}^{\tau}\right) \neq 0$. Since $\hat{P}_{N} \hat{B}=\hat{B} \hat{P}_{N}$,

$$
\hat{A}_{N}^{\tau}=\hat{B}_{N}^{\tau}\left(\hat{S}_{N}^{1, \tau}-\gamma \hat{I}_{N}\right)
$$


which means that $\hat{B}_{N}^{T}\left(\hat{S}_{N}^{1, \tau}-\gamma \hat{I}_{N}\right)$ has trivial kernel. Since $\hat{B}_{N}$ has trivial kernel, we know that

$$
\operatorname{ker}\left(\hat{S}_{N}^{1, \tau}-\gamma \hat{I}_{N}\right)=\{0\}
$$

and therefore $\gamma \in \rho\left(\hat{S}_{N}^{1, \tau}-\gamma \hat{I}_{N}\right)$ for $N \geq M_{\gamma}$.

Given this theorem, we prove the following corollary.

Corollary 8. If $\lambda_{N_{j}} \in \sigma\left(\hat{S}_{N_{j}}^{1, \tau}\right)$ and $\lambda_{N_{j}} \rightarrow \gamma$, then $\gamma \in \sigma\left(S^{1}\right)$.

Proof. Suppose in contradiction that $\gamma \in \rho\left(S^{1}\right)$. Then, by Theorem 7 we know for some value $M$ that $\gamma \in \rho\left(\hat{S}_{N}^{1, \tau}\right)$ for $N \geq M$. Then

$$
\begin{aligned}
\left\|\left(\hat{S}_{N}^{1, \tau}-\gamma\right)^{-1}\right\|_{2} & =\left\|\left(\hat{B}_{N}^{\tau}\left(\hat{S}_{N}^{1, \tau}-\gamma\right)\right)^{-1} \hat{B}_{N}^{\tau}\right\|_{2} \\
& \leq\left\|\left(\hat{B}_{N}^{\tau}\left(\hat{S}_{N}^{1, \tau}-\gamma\right)\right)^{-1}\right\|_{2}\left\|\hat{B}_{N}^{\tau}\right\|_{2} \\
& \leq\left\|\left(\hat{B}_{N}^{\tau}\left(\hat{S}_{N}^{1, \tau}-\gamma\right)\right)^{-1}\right\|_{2}\|\hat{B}\|_{2} .
\end{aligned}
$$

Following the notation in Theorem [7. $\hat{B}_{N}^{T}\left(\hat{S}_{N}^{1, \tau}-\gamma\right)=\hat{I}_{N}+\hat{K}_{N}^{\tau}$. Likewise, per our convention, let $\hat{K}_{N}$ denote the $l_{2}$ operator such that $\hat{K}_{N}^{\tau}$ is the $(2 N+1) \times(2 N+1)$ truncation of $\hat{K}_{N}$, and $\hat{K}_{N}=\hat{P}_{N} \hat{K}_{N} \hat{P}_{N}=\hat{P}_{N} \hat{K} \hat{P}_{N}$. From Theorem 7 , we know that $K$ is compact, and therefore $\hat{K}_{N}$ converges to $K$ in the uniform operator topology. Clearly

$$
\left\|\left(\hat{I}_{N}^{\tau}+\hat{K}_{N}^{\tau}\right)^{-1}\right\|_{2} \leq\left\|\left(\hat{I}+\hat{K}_{N}\right)^{-1}\right\|_{2},
$$

and we know that $I+K$ has a bounded inverse. This implies that

$$
\left\|\left(\hat{I}+\hat{K}_{N}\right)^{-1}\right\|_{2} \leq\left\|(\hat{I}+\hat{K})^{-1}\right\|_{2}\left\|\left(\hat{I}+(\hat{I}+\hat{K})^{-1}\left(\hat{K}_{N}-\hat{K}\right)\right)^{-1}\right\|_{2} .
$$

Since $\hat{K}_{N}$ converges uniformly to $\hat{K}$, there exists $L$ such that $\left\|(\hat{I}+\hat{K})^{-1}\left(\hat{K}_{N}-\hat{K}\right)\right\|$ $<1 / 2$ for $N \geq L$, and therefore

$$
\left\|\left(\hat{I}+(\hat{I}+\hat{K})^{-1}\left(\hat{K}_{N}-\hat{K}\right)\right)^{-1}\right\|_{2} \leq 2 .
$$

Finally, we know that

$$
\left\|\left(\hat{S}_{N}^{1, \tau}-\gamma\right)^{-1}\right\|_{2} \geq \frac{1}{d\left(\gamma, \sigma\left(\hat{S}_{N}^{1, \tau}\right)\right)},
$$

where

$$
d\left(\gamma, \sigma\left(\hat{S}_{N}^{1, \tau}\right)\right)=\inf _{s \in \sigma\left(\hat{S}_{N}^{1, \tau}\right)}|\gamma-s|
$$

This implies that

$$
d\left(\gamma, \sigma\left(\hat{S}_{N}^{1, \tau}\right)\right) \geq \frac{2}{\|\hat{B}\|_{2}\left\|(\hat{I}+\hat{K})^{-1}\right\|_{2}}
$$

for $N \geq S$. Hence, if $\gamma \in \rho\left(S^{1}\right)$, there can be no subsequence $\lambda_{N_{j}} \in \sigma\left(\hat{S}_{N_{j}}^{1, \tau}\right)$ converging to $\gamma$.

Now we can prove the restricted no-spurious-mode condition. 
Theorem 9. Let $D$ be some compact set in the complex plane, and let $\left\{\lambda_{N}\right\}_{N=1}^{\infty}$ be a sequence contained in $D$ with $\lambda_{N} \in \sigma\left(\hat{S}_{N}^{1, \tau}\right)$. Then for all $\epsilon>0$, there exists some integer $M$ such that $\lambda_{N}$ is in an $\epsilon$-neighborhood of some value $\lambda \in D \cap \sigma\left(S^{1}\right)$ for $N \geq M$.

Proof. Suppose instead that there exists a subsequence $\lambda_{N_{j}}$ such that $d\left(\lambda_{N_{j}}, D \cap\right.$ $\left.\sigma\left(S^{1}\right)\right) \geq \epsilon>0$. However, since $D$ is compact, $\lambda_{N_{j}}$ must have a convergent subsequence, and this subsequence must converge to some element in $\sigma\left(S^{1}\right)$ by Corollary 8. Hence our original assumption cannot hold, and the theorem is proved.

3.2. Proof of the second condition. We were able to prove the first condition under quite general assumptions. Specifically, it was not necessary to impose that $S^{1}$ was a selfadjoint operator. We are unable to prove the second condition without making this assumption. However, it should be noted that for non-selfadjoint operators, we have been unable to find numerical examples where the second condition appears not to hold.

Our proof relies on a number of results from [18. To apply these, we need the following lemma.

Lemma 10. $P_{N} S^{1} P_{N}$ converges strongly to $S^{1}$.

Proof. Let $\psi \in \mathrm{H}^{p}\left(\mathrm{~S}_{T}\right)$. Then

$$
\begin{aligned}
\left\|S^{1} \psi-P_{N} S^{1} P_{N} \psi\right\|_{2} & =\left\|P_{N} S^{1}\left(\mathrm{I}-P_{N}\right) \psi+\left(\mathrm{I}-P_{N}\right) S^{1} \psi\right\|_{2} \\
& \leq\left\|S^{1}\left(\mathrm{I}-P_{N}\right) \psi\right\|_{2}+\left\|\left(\mathrm{I}-P_{N}\right) S^{1} \psi\right\|_{2} \\
& \leq C\left\|\left(\mathrm{I}-P_{N}\right) \psi\right\|_{2, p}+\left\|\left(\mathrm{I}-P_{N}\right) S^{1} \psi\right\|_{2} .
\end{aligned}
$$

This must become arbitrarily small as $N \rightarrow \infty$. Therefore the lemma is proved.

The results we need from [18] will now be stated for the sake of completeness. Proofs of the lemmas and theorem can be found in [18, pages 290-292.

Definition 11. For any linear operator $T$, if $\gamma \in \rho(T)$, the resolvent operator of $T$ is defined as

$$
\mathrm{R}_{\gamma}(T) \equiv(T-\gamma)^{-1} .
$$

Lemma 12. If $T$ is a selfadjoint operator, then

$$
\left\|R_{\gamma}(T)\right\|_{2}=\frac{1}{d(\gamma, \sigma(T))}
$$

where $d(\gamma, \sigma(T))=\inf _{s \in \sigma(T)}|\gamma-s|$.

Lemma 13. If $T$ is selfadjoint and $\operatorname{Im}(\gamma) \neq 0$, then

$$
\left\|R_{\gamma}(T)\right\|_{2} \leq \frac{1}{|\operatorname{Im}(\gamma)|}
$$

Definition 14. Given a linear operator $T$ with domain $\mathrm{D}(T)$, a core of $T$ is a subset $\mathrm{D} \subset \mathrm{D}(T)$ such that

$$
\overline{\left.T\right|_{D}}=T,
$$

where $\overline{\left.T\right|_{D}}$ is the smallest closed extension of $\left.T\right|_{D}$. 
Our operator $S^{1}$ is closed over $\mathrm{H}^{p}\left(\mathrm{~S}_{T}\right)$ [15]. Therefore $\mathrm{H}^{p}\left(\mathrm{~S}_{T}\right)$ is a core for $S^{1}$. Likewise, each of the finite-rank operators $P_{N} S^{1} P_{N}$ is continuous, and consequently, closed on $\mathrm{H}^{p}\left(\mathrm{~S}_{T}\right)$. This makes $\mathrm{H}^{p}\left(\mathrm{~S}_{T}\right)$ a common core for $S^{1}$ and $P_{N} S^{1} P_{N}$. We can then use

Lemma 15. Let $P_{N} S^{1} P_{N}$ and $S^{1}$ be selfadjoint operators on a common core $D$. If $P_{N} S^{1} P_{N}$ converges strongly to $S^{1}$ on $D$, then $R_{\gamma}\left(P_{N} S^{1} P_{N}\right)$ converges strongly to $R_{\gamma}\left(S^{1}\right)$ if $\operatorname{Im}(\gamma) \neq 0$.

Finally, given the above lemma, we use the following theorem.

Theorem 16. Let $P_{N} S^{1} P_{N}$ and $S^{1}$ be selfadjoint on a common core $D$. If $R_{\gamma}\left(P_{N} S^{1} P_{N}\right)$ converges strongly to $R_{\gamma}\left(S^{1}\right)$ for $\operatorname{Im}(\gamma) \neq 0$, and if $a<b$ and $(a, b) \subset \rho\left(P_{N} S^{1} P_{N}\right)$ for $N$ sufficiently large, then $(a, b) \subset \rho\left(S^{1}\right)$.

Proof. See [18], page 290.

Theorem 16 can be modified to accommodate subsequences, since the strong convergence of $P_{N} S^{1} P_{N}$ to $S^{1}$ also holds for subsequences. This lets us prove the second condition. Suppose the second condition were false. This implies that there exists $\lambda \in \sigma\left(S^{1}\right)$ such that

$$
d\left(\lambda, \sigma\left(\hat{S}_{N_{j}}^{1, \tau}\right)\right) \geq \epsilon
$$

for $j$ sufficiently large. Suppose further that $\lambda \neq 0$. This implies that the disc $B_{\lambda}(\epsilon)=\{z \in \mathbb{C}:|z-\lambda|<\epsilon\}$ is a subset of $\rho\left(\hat{S}_{N_{j}}^{1, \tau}\right)$, which implies that $B_{\lambda}(\tilde{\epsilon}) \subset$ $\rho\left(P_{N} S^{1} P_{N}\right)$, where $\tilde{\epsilon} \leq \epsilon$. Therefore, by Theorem 16, $B_{\lambda}(\tilde{\epsilon}) \subset \rho\left(S^{1}\right)$. This is a contradiction, which implies the second condition for $\lambda \neq 0$. If $\lambda=0$, we need only pick some $c \in \rho\left(S^{1}\right)$ and repeat our steps for $S^{1}-c$.

\section{Convergence of eigenfunctions}

We assume in advance that the approximate eigenvalues, $\lambda_{N} \in \sigma\left(\hat{S}_{N}^{1, \tau}\right)$, converge to some $\lambda \in \sigma\left(S^{1}\right)$. Given $\lambda_{N} \in \sigma\left(\hat{S}_{N}^{1, \tau}\right)$, there exists a $(2 N+1)$-dimensional vector $\hat{\phi}_{N}^{\tau}$ such that

$$
\hat{S}_{N}^{1, \tau} \hat{\phi}_{N}^{\tau}=\lambda_{N} \hat{\phi}_{N}^{\tau},\left\|\hat{\phi}_{N}^{\tau}\right\|_{2}=1
$$

We prove the following proposition.

Theorem 17. If $\lambda_{N} \in \sigma\left(\hat{S}_{N}^{1, \tau}\right)$ converges to $\lambda \in \sigma\left(S^{1}\right)$, then there exists a vector $\hat{\phi}$ such that a subsequence of $\hat{\phi}_{N}$ converges to $\hat{\phi}$ in $\|\cdot\|_{2}$ and $S^{1} \phi=\lambda \phi$.

Proof. We extend the vectors $\hat{\phi}_{N}^{\tau}$ to vectors $\hat{\phi}_{N}$ so that $\hat{P}_{N} \hat{\phi}_{N}=\hat{\phi}_{N}$. Given that $\left\|\hat{\phi}_{N}\right\|_{2}=1$, by Alaoglu's theorem [14 there exists a vector $\hat{\phi}$ such that some subsequence of $\hat{\phi}_{N}$, denoted as $\hat{\phi}_{N}$, converges weakly to $\hat{\phi}$. Using the operator $B$ from the proof of Theorem [7, and noting that $B$ commutes with the projection operator $P_{N}$, we get

$$
\begin{aligned}
\lambda_{N} \hat{B} \hat{\phi}_{N} & =\hat{B} \hat{P}_{N} \hat{S}^{1} \hat{P}_{N} \hat{\phi}_{N} \\
& =\hat{P}_{N} \hat{B} \hat{S}^{1} \hat{\phi}_{N} \\
& =\hat{P}_{N}(\hat{I}+\hat{K}) \hat{\phi}_{N} \\
& =\hat{\phi}_{N}+\hat{P}_{N} \hat{K} \phi_{N} .
\end{aligned}
$$


$B$ is a compact operator since

$$
\begin{aligned}
\left\|\left(\hat{B} \hat{P}_{N}-\hat{B}\right) \hat{\psi}\right\|_{2}^{2} & \leq \sum_{|n|>N}\left(\frac{2 \pi n}{T}\right)^{-2 p}\left|\hat{\psi}_{n}\right|^{2} \\
& \leq\left(\frac{2 \pi N}{T}\right)^{-2 p}\|\hat{\psi}\|_{2}^{2} .
\end{aligned}
$$

This implies that $\left\|\hat{B} \hat{P}_{N}-\hat{B}\right\|_{2} \leq\left(\frac{2 \pi N}{T}\right)^{-p}$, and so $B$ is a uniform limit of finiterank operators and is therefore compact. $B$ compact then implies that $\hat{B} \hat{\phi}_{N} \rightarrow \hat{B} \hat{\phi}$. Likewise, since $K$ is compact, we have $\hat{K} \hat{\phi}_{N} \rightarrow \hat{K} \hat{\phi}, \hat{P}_{N} \hat{K} \rightarrow \hat{K}$ uniformly, and

$$
\left\|\hat{P}_{N} \hat{K} \hat{\phi}_{N}-\hat{K} \hat{\phi}\right\|_{2} \leq\left\|\hat{K}_{\phi_{N}}-\hat{K} \hat{\phi}\right\|_{2}+\left\|\hat{P}_{N} \hat{K}-\hat{K}\right\|_{2}\|\hat{\phi}\|_{2}
$$

which implies $\hat{P}_{N} \hat{K} \hat{\phi}_{N} \rightarrow \hat{K} \hat{\phi}$. Therefore, we have

$$
\hat{\phi}_{N} \rightarrow \lambda \hat{B} \hat{\phi}-\hat{K} \hat{\phi} \text {. }
$$

Our weakly convergent sequence $\hat{\phi}_{N}$ has been shown to converge strongly. This implies that

$$
\hat{\phi}_{N} \rightarrow \hat{\phi},
$$

and using (75),

$$
(\hat{I}+\hat{K}) \hat{\phi}=\lambda \hat{B} \hat{\phi} .
$$

We still need to show that the function $\phi$, corresponding to the symbol $\hat{\phi}$, is in $\mathrm{D}\left(S^{1}\right)$, and that it is an eigenfunction. There are two cases to consider. The first is $\lambda \neq 0$. This implies that $\hat{S}^{1}$ is invertible, and we showed in Theorem 7 that the operator $\hat{I}+\hat{K}$ is invertible. If $\hat{I}+\hat{K}$ is invertible, then

$$
(\hat{I}+\hat{K})^{-1}\left(\widehat{B S^{1}}\right)=\hat{I}
$$

where $\widehat{B S^{1}}$ denotes the extension of $\hat{B} \hat{S}^{1}$. Likewise, if $S^{1}$ is invertible, then for $\phi \in \mathrm{L}_{2}\left(\mathrm{~S}_{T}\right)$ there must exist some $\psi \in \mathrm{H}^{p}\left(\mathrm{~S}_{T}\right)$, with symbol $\hat{\psi}$, such that $\hat{S}^{1} \hat{\psi}=\hat{\phi}$. This implies that

$$
\begin{aligned}
\hat{\phi} & =\lambda(\hat{I}+\hat{K})^{-1} \hat{B} \hat{\phi} \\
& =\lambda(\hat{I}+\hat{K})^{-1} \hat{B} \hat{S}^{1} \hat{\psi} \\
& =\lambda(\hat{I}+\hat{K})^{-1} \widehat{B S^{1}} \hat{\psi} \\
& =\lambda \hat{\psi},
\end{aligned}
$$

and therefore $\phi$ is an eigenfunction of $S^{1}$.

The second case to consider is $\lambda=0$. In that case let $c \in \rho\left(S^{1}\right)$ so that the operator $S^{1}-c$ is invertible. Repeat the steps for the $\lambda \neq 0$ case.

If we assume that the eigenvalue $\lambda$ is simple, then we see that every subsequence of $\phi_{N}$ converges to some unit multiple of $\phi$ since we claimed every sequence of approximate eigenvectors $\phi_{N}$ has a convergent subsequence. We can then say, upon appropriate rescalings, that the sequence is convergent. The general problem for non-simple eigenvalues appears to be rather difficult, and we do not address it here. 


\section{Rate of CONVERGENCE}

Before proceeding, we need two technical lemmas. The first lemma is from [1], page 69 . We include the proof for clarity.

Lemma 18. If $\phi \in \mathbf{C}^{\infty}\left(S_{T}\right)$, then $\left\|\left(I-P_{N}\right) \phi\right\|_{2}=O\left(N^{-p}\right)$ for all integer values of $p>0$.

Proof. Since $\mathbf{C}^{\infty}\left(S_{T}\right) \subset \mathrm{H}^{p}\left(\mathrm{~S}_{T}\right)$ for arbitrary $p$, we can write

$$
\begin{aligned}
\left\|\left(I-P_{N}\right) \phi\right\|_{2}^{2} & =\sum_{|n|>N}\left|\hat{\phi}_{n}\right|^{2} \\
& =\left(\frac{T}{2 \pi N}\right)^{2 p} \sum_{|n|>N}\left(\frac{2 \pi N}{T}\right)^{2 p}\left|\hat{\phi}_{n}\right|^{2} \\
& \leq\left(\frac{T}{2 \pi N}\right)^{2 p} \sum_{|n|>N}\left(\frac{2 \pi n}{T}\right)^{2 p}\left|\hat{\phi}_{n}\right|^{2} \\
& \leq\left(\frac{T}{2 \pi N}\right)^{2 p}\|\phi\|_{2, p}^{2} .
\end{aligned}
$$

Therefore

$$
\left\|\left(I-P_{N}\right) \phi\right\|_{2}=\mathrm{O}\left(N^{-p}\right), p>0 .
$$

The second lemma relies upon a restriction of the selfadjoint operator $S^{1}$ to the form

$$
S^{1}=\partial_{x}^{p}+\sum_{k=1}^{p-1} c_{k} \partial_{x}^{k}+f(x),
$$

where the $c_{i}$ are constants. We denote the constant-coefficient differential operator as $D_{p}^{\mu}$. This restriction greatly simplifies our work since the operator $P_{N}$ commutes with $D_{p}^{\mu}$. We now prove our second technical lemma:

Lemma 19. If $\phi_{N}$ and $\phi$ are the approximate and true eigenfunctions, respectively, of the operator $D_{p}^{\mu}+f(x)$, then convergence in $\|\cdot\|_{2}$ implies convergence in $\|\cdot\|_{2, k}$ for all positive integers $k$.

Proof. We have $P_{N} S^{1} \phi_{N}=D_{p}^{\mu} \phi_{N}+P_{N} f(x) \phi_{N}$. As shown in the previous section, if approximate eigenfunctions $\phi_{N}$ converge to $\phi$ in the $\|\cdot\|_{2}$ norm, then

$$
\lim _{N \rightarrow \infty}\left\|P_{N} f(x) \phi_{N}-f(x) \phi\right\|_{2}=0 .
$$

This implies that

$$
\lim _{N \rightarrow \infty}\left\|D_{p}^{\mu} \phi_{N}-D_{p}^{\mu} \phi\right\|_{2}=0
$$

which means that $\phi_{N}$ converges to $\phi$ in the graph norm associated with $S^{1}$, i.e.

$$
\lim _{N \rightarrow \infty}\left(\left\|\phi_{N}-\phi\right\|_{2}+\left\|S^{1} \phi_{N}-S^{1} \phi\right\|_{2}\right)=0 .
$$

The graph norm associated with the operator $S^{1}$ is equivalent to the $p^{\text {th }}$ Sobolev norm [15], which implies that

$$
\lim _{N \rightarrow \infty}\left\|\phi_{N}-\phi\right\|_{2, p}=0 .
$$


Convergence in $\|\cdot\|_{2, p}$ implies $\partial_{x} \phi_{N} \rightarrow \partial_{x} \phi$ in $\|\cdot\|_{2}$, and thus $\partial_{x}\left(D_{p}^{\mu} \phi_{N}+f(x) \phi_{N}\right)$ converges to $\partial_{x} S^{1} \phi$ in $\|\cdot\|_{2}$. This implies that $\phi_{N}$ converges to $\phi$ in $\|\cdot\|_{2, p+1}$. Proceeding this way, we see that $\phi_{N}$ converges to $\phi$ in $\|\cdot\|_{2, k}$ for all integers $k>$ 0.

Finally, we need the following min-max theorem [11]:

Theorem 20. Suppose the selfadjoint operator $S^{1}$ has least eigenvalue $\lambda_{0}>-\infty$. Then

$$
\lambda_{0}=\inf _{\|\psi\|_{2}=1}\left\langle S^{1} \psi, \psi\right\rangle,
$$

where $\psi$ is understood to be in the domain of $S^{1}$.

Using Theorem 20 and our technical lemmas, we prove the next theorem.

Theorem 21. Let $\lambda=\min \sigma\left(S^{1}\right)>-\infty, \lambda$ simple. Then there exists a sequence $\lambda_{N} \rightarrow \lambda, \lambda_{N} \in \sigma\left(\hat{S}_{N}^{1, \tau}\right)$, and

$$
\left|\lambda_{N}-\lambda\right|=O\left(N^{-q}\right), q \geq 1 .
$$

Proof. By Theorem 20, we have

$$
\lambda=\inf _{\|\psi\|_{2}=1}\left\langle S^{1} \psi, \psi\right\rangle .
$$

Define the sequence $\left\{\lambda_{N}\right\}_{N=1}^{\infty}$ via

$$
\lambda_{N}=\inf _{\left\|\hat{\psi}_{N}^{\tau}\right\|_{2}=1}\left\langle\hat{S}_{N}^{1, \tau} \hat{\psi}_{N}^{\tau}, \hat{\psi}_{N}^{\tau}\right\rangle
$$

where $\hat{\psi}_{N}^{\tau} \in \mathbb{C}^{2 N+1}$. Let $\hat{\psi}_{N}$ denote the extension of $\hat{\psi}_{N}^{\tau}$, i.e. $\hat{P}_{N} \hat{\psi}_{N}=\hat{\psi}_{N}$. We can equivalently define $\lambda_{N}$ as

$$
\lambda_{N}=\inf _{\left\|\psi_{N}\right\|_{2}=1}\left\langle S^{1} \psi_{N}, \psi_{N}\right\rangle .
$$

This shows that $\lambda_{N} \geq \lambda_{N+1} \geq \cdots \geq \lambda$. Since the $\lambda_{N}$ 's are a monotone sequence, they must have a limit, say $\tilde{\lambda}$. Suppose $\tilde{\lambda}>\lambda$. Since we know that $\lambda \in \sigma\left(S^{1}\right)$ and that $S^{1}$ is selfadjoint, by our second condition for convergence, we know there exists a sequence $\gamma_{N} \in \sigma\left(\hat{S}_{N}^{1, \tau}\right)$ such that $\gamma_{N} \rightarrow \lambda$. This implies for $N$ large enough that $\gamma_{N}<\tilde{\lambda}$, but this would imply that $\gamma_{N}<\lambda_{N}$, which is impossible. Therefore $\tilde{\lambda}=\lambda$ and $\lambda_{N} \rightarrow \lambda$.

Let $c>\lambda_{1}, c \neq 0$. We can alter our definition of $\lambda_{N}$ to

$$
\lambda_{N}=\inf _{\|\psi\|_{2}=1}\left(\left\langle S^{1} P_{N} \psi, P_{N} \psi\right\rangle+c\left\langle\left(I-P_{N}\right) \psi, \psi\right\rangle\right) .
$$

We introduce this alteration in order to take infimums over the same domain. Let each eigenvalue $\lambda_{N}$ have corresponding eigenvector $\phi_{N}$, and let $\lambda$ have eigenvector $\phi$. We showed in Section 4 that $\phi_{N} \rightarrow \phi$ in $\|\cdot\|_{2}$, so we can restrict ourselves to the set

$$
E_{N}=\left\{\phi_{j}\right\}_{j \geq N}
$$

Assume that $\lambda>0$, which implies $\lambda_{N}>0$. Consider the difference

$$
\frac{1}{\lambda}-\frac{1}{\lambda_{N}}=\sup _{E_{N}} \frac{1}{\left\langle S^{1} \psi, \psi\right\rangle}-\sup _{E_{N}} \frac{1}{\left(\left\langle S^{1} P_{N} \psi, P_{N} \psi\right\rangle+c\left\langle\left(I-P_{N}\right) \psi, \psi\right\rangle\right)} .
$$


We have

$$
\begin{array}{r}
\left\langle S^{1} \psi, \psi\right\rangle=\left\langle S^{1} P_{N} \psi, P_{N} \psi\right\rangle+R_{N}(\psi), \\
R_{N}(\psi) \equiv\left\langle\left(I-P_{N}\right) \psi, S^{1} P_{N} \psi\right\rangle+\left\langle S^{1} \psi,\left(I-P_{N}\right) \psi\right\rangle,
\end{array}
$$

and so

$$
\frac{1}{\lambda}-\frac{1}{\lambda_{N}} \leq \frac{1}{\lambda \lambda_{N}} \sup _{E_{N}}\left|-R_{N}(\psi)+c\left\langle\left(I-P_{N}\right) \psi, \psi\right\rangle\right|
$$

or

$$
\lambda_{N}-\lambda \leq \sup _{E_{N}}\left|-R_{N}(\psi)+c\left\langle\left(I-P_{N}\right) \psi, \psi\right\rangle\right| .
$$

Using Cauchy-Schwarz,

$$
\begin{aligned}
\left|R_{N}(\psi)\right| & \leq\left(\left\|S^{1} P_{N} \psi\right\|_{2}+\left\|S^{1} \psi\right\|_{2}\right)\left\|\left(I-P_{N}\right) \psi\right\|_{2}, \\
\left|c\left\langle\left(I-P_{N}\right) \psi, \psi\right\rangle\right| & \leq c\|\psi\|_{2}\left\|\left(I-P_{N}\right) \psi\right\|_{2} .
\end{aligned}
$$

Given the result of Lemma19, we bound $\left(\left\|S^{1} P_{N} \psi\right\|_{2}+\left\|S^{1} \psi\right\|_{2}\right)$ by some constant $M$. Since $E_{N}$ is closed, there must be some vector $\phi_{K} \in E_{N}$ such that

$$
\sup _{E_{N}}\left|-R_{N}(\psi)+c\left\langle\left(I-P_{N}\right) \psi, \psi\right\rangle\right| \leq(M+c)\left\|\left(I-P_{N}\right) \phi_{K}\right\|_{2} .
$$

Knowing that each $\phi_{K}$ is smooth, Lemma 18 implies

$$
\lambda_{N}-\lambda \leq(M+c)\left(\frac{T}{2 \pi N}\right)^{q}\left\|\phi_{K}\right\|_{2, q}
$$

for all $q>0$. Lemma 19 shows that $\left\|\phi_{K}\right\|_{2, q} \rightarrow\|\psi\|_{2, q}$. So, for a given $\epsilon$, there must be some value $L$ such that $\left\|\phi_{K}\right\|_{2, q} \leq(1+\epsilon)\|\psi\|_{2, q}$ for all $K \geq L$. Hence, for $N \geq L$, we have

$$
\lambda_{N}-\lambda \leq(M+c)(1+\epsilon)\left(\frac{T}{2 \pi N}\right)^{q}\|\psi\|_{2, q} .
$$

In the case that $\lambda \leq 0$, pick $\alpha$ such that $\alpha+\lambda>0$. Likewise we see that

$$
\begin{aligned}
\alpha+\lambda & =\inf _{\|\psi\|_{2}=1}\left\langle\left(S^{1}+\alpha\right) \psi, \psi\right\rangle, \\
\alpha+\lambda_{N} & =\inf _{\left\|\psi_{N}\right\|_{2}=1}\left\langle\left(S^{1}+\alpha\right) \psi_{N}, \psi_{N}\right\rangle .
\end{aligned}
$$

Then we repeat our argument from above.

Note, in the case that $\inf \sigma\left(S^{1}\right)=-\infty$, but $\sup \sigma\left(S^{1}\right)<\infty$, we can apply the theorem just proved to the operator $-S^{1}$.

\section{ACKNOWLEDGEMENTS}

We wish to express our gratitude to the referees for bringing a number of interesting references to our attention, especially [13. We feel this reference deserves broader and more popular recognition than it seems to have received thus far. This work was supported by the National Science Foundation through grants NSFDMS-0604546 (BD) and NSF-DMS-VIGRE-0354131(CC). Any opinions, findings, and conclusions or recommendations expressed in this material are those of the authors and do not necessarily reflect the views of the funding sources. 


\section{REFERENCES}

1. K. Atkinson, The numerical solution of integral equations of the second kind, Cambridge University Press, Cambridge, 1997. MR 1464941 (99d:65364)

2. K. Atkinson and W. Han, Theoretical numerical analysis: A functional analysis framework, Springer, New York, NY, 2005. MR2153422(2006a:65001)

3. A. Böttcher, Infinite matrices and projection methods, Lectures on Operator Theory and Its Applications, American Mathematical Society, Providence, RI, 1996, pp. 3-72. MR 1364444 (96m:47016)

4. A. Böttcher and B. Silbermann, Analysis of Toeplitz operators, Springer-Verlag, New York, NY, 1990. MR.1071374 (92e:47001)

5. J.B. Conway, A course in functional analysis, 2nd ed., Springer, New York, NY, 1990. MR.1070713 (91e:46001)

6. B. Deconinck and J. N. Kutz, Computing spectra of linear operators using the Floquet-FourierHill method, J. Comput. Phys. 219 (2006), 296-321. MR.2273379 (2007g:37078)

7. L.M. Delves and T.L. Freeman, Analysis of global expansion methods: Weakly asymptotically diagonal systems, Academic Press, New York, NY, 1981. MR624434 (84a:65002)

8. I. Gohberg, S. Goldberg, and M.A. Kaashoek, Classes of linear operators: Vol. I, BirkhäuserVerlag, Basel, 1990. MR.1130394 (93d:47002)

9. I. Gohberg, S. Goldberg, and N. Krupnick, Traces and determinants of linear operators, Birkhäuser-Verlag, Basel, 2000. MR1744872 (2001b:47035)

10. G. W. Hill, On the part of the motion of the lunar perigee which is a function of the mean motions of the sun and moon, Acta Math. 8 (1886), no. 1, 1-36. MR 1554690

11. P.D. Hislop and I.M. Sigal, Introduction to spectral theory: With applications to Schrödinger operators, Springer, New York, NY, 1996. MR1361167 (98h:47003)

12. T. Kato, Perturbation theory for linear operators, 2nd ed., Springer-Verlag, New York, N.Y., 1976. MR0407617 (53:11389)

13. M.A. Krasnosel'skiı̌, G.M. Vainikko, P.P. Zabreiko, Ya. B. Rutitskii, and V. Ya. Stetsenko, Approximate solution of operator equations, Wolters-Noordhoff, Groningen, 1972. MR 0385655 $(52: 6515)$

14. P.D. Lax, Functional analysis, Wiley, New York, NY, 2002. MR1892228 (2003a:47001)

15. J. Locker, Functional analysis and two-point differential operators, Longman Scientific and Technical, New York, NY, 1986. MR865984 (88d:34001)

16. A.W. Naylor and G.R. Sell, Linear operator theory in engineering and science, SpringerVerlag, New York, NY, 1982. MR672108 (83j:46001)

17. H. Poincaré, Sur les déterminants d'ordre infini, Bull. Soc. Math. France 14 (1886), 77-90. MR 1503979

18. M. Reed and B. Simon, Methods of modern mathematical physics. I. Functional analysis, Academic Press, New York, NY, 1972. MR0493419 (58:12429a)

19. H. Sandberg, E. Mollerstedt, and B. Bernhardsson, Frequency-domain analysis of linear time-periodic systems, IEEE Trans. of Auto. Control 50 (2005), 1971-1983. MR2186267 (2006h:93067)

20. B. Sandstede, Stability of travelling waves, Handbook of Dynamical Systems, Vol. 2 (B. Fiedler, ed.), Elsevier Science B.V., 2002, pp. 983-1055. MR1901069 (2004e:37121)

21. M. Schechter, Principles of functional analysis, 2nd ed., American Mathematical Society, Providence, RI, 2001. MR1861991 (2002j:46001)

22. H. von Koch, Sur les systèmes d'une infinité d'équations linéaires à une infinité d'inconnues, C. R. Cong. d. Math. Stockholm (1910), 43-61.

23. J. Zhou, Zeros and poles of linear continuous-time periodic systems: Definitions and properties, IEEE Trans. of Auto. Control 53 (2008), 1998-2011. MR2459573

24. J. Zhou, T. Hagiwara, and M. Araki, Spectral characteristics and eigenvalues computation of the harmonic state operators in continuous-time periodic systems, Systems and Control Letters 53 (2004), 141-155. MR2091839(2005k:93047)

Department of Applied Mathematics, University of Washington, Seattle, WashingTON 98195-3420

E-mail address: curtchr@amath.washington.edu

Department of Applied Mathematics, University of Washington, Seattle, WashingTON 98195-3420 